\title{
Ocular ultrasonography in patients with subarachnoid hemorrhage and Terson syndrome
}

\author{
Livio Vitiello, MD • Maddalena De Bernardo, MD, PhD (1) Nicola Rosa, MD
}

Received: 2 January 2020/Revised: 7 January 2020/Accepted: 15 February 2020/Published online: 9 March 2020

(C) Canadian Anesthesiologists' Society 2020

\section{To the Editor,}

We read the interesting case series by Palermo et al. concerning ocular ultrasonography in two patients with subarachnoid hemorrhage and Terson syndrome. ${ }^{1}$ We would like to comment on some aspects of B-scan ultrasonography to evaluate the optic nerve sheath diameter (ONSD). This ultrasound method is gaining in popularity for its accessibility, low-cost, and safety profile, with wide clinical utility in different medical fields.

However, it has been shown to be quite inaccurate in assessing small structures, such as the ONSD, because it does not permit the operator to obtain objective and exact measurements because of, among other factors, the blooming effect. This effect implies a reduction in the size of a structure just by increasing the gain of the B-scan ultrasonography, whereas the same structure will appear larger when the gain is reduced. ${ }^{2,3}$

In this case, as can be observed in Fig. 1 of the cited report, ${ }^{1}$ the three pictures seem to have been measured at three different time points during the follow up. In fact, it appears that the optic nerve was not caught at its insertion, as the elevation of the optic disc is not evident in the picture, as it should be in case of papilledema.

For these reasons, clinicians should consider using the standardized A-scan technique, which is a blooming effectfree ultrasonography modality that requires an $8 \mathrm{MHz}$

This letter is accompanied by a reply. Please see Can J Anesth 2020; 67: this issue.

L. Vitiello, MD · M. De Bernardo, MD, PhD ( $₫)$.

N. Rosa, MD

Department of Medicine, Surgery, and Dentistry, Scuola Medica

Salernitana, University of Salerno, Salerno, Italy

e-mail: mdebernardo@unisa.it unfocused transducer to obtain spikes instead of dots. This technique could potentially provide more accurate ONSD measurements. ${ }^{4}$ Furthermore, when coupled with the $30^{\circ}$ test, which consists of measuring the maximal optic nerve diameter first in straight gaze ("0 degrees") and then in abduction ("30 degrees"), this ultrasound method can show an increase in subarachnoidal fluid when the ONSD decreases greater than 5\% during maximal eye abduction. This can also differentiate an increase in ONSD due to intracranial hypertension from a solid thickening of pial and arachnoidal sheaths related to optic neuritis, optic nerve glioma, meningioma, or leukemic infiltration. ${ }^{5}$ Unfortunately, this is a more difficult exam, which requires some additional training.

In conclusion, we would like to suggest the use of Ascan in further studies regarding the ultrasonographic assessment of the ONSD, with the hopes that this method would offer more replicable and objective results.

Conflict of interest None.

Financial statement None.

Editorial responsibility This submission was handled by Dr. Philip M. Jones, Associate Editor, Canadian Journal of Anesthseia.

\section{References}

1. Palermo J, Bojanowski M, Langevin S, Denault AY. Point-of-care handheld ophthalmic ultrasound in the diagnosis and evaluation of raised intracranial pressure and Terson syndrome: a description of two cases. Can J Anesth 2019. DOI: https://doi.org/10.1007/ s12630-019-01531-4.

2. De Bernardo $M$, Vitiello L, Rosa $N$. Ocular ultrasound assessment to estimate the risk of increased intracranial pressure after 
traumatic brain injury in prehospital setting. Prehosp Emerg Care 2019; 23: 746-7.

3. Rosa N, Vitiello L, De Bernardo M. Optic nerve sheath diameter measurement in hypoxic ischaemic brain injury after cardiac arrest. Resuscitation 2019; 138: 310-1.

4. De Bernardo $M$, Vitiello L, Rosa N. Intracranial pressure evaluation in acute liver failure. Neurocrit Care 2019; 30: 495-6.
5. De Bernardo $M$, Vitiello L, Rosa N. Optic nerve ultrasonography to predict increased intracranial pressure in idiopathic intracranial hypertension. Neuroradiol J 2019; 32: 227-8.

Publisher's Note Springer Nature remains neutral with regard to jurisdictional claims in published maps and institutional affiliations. 